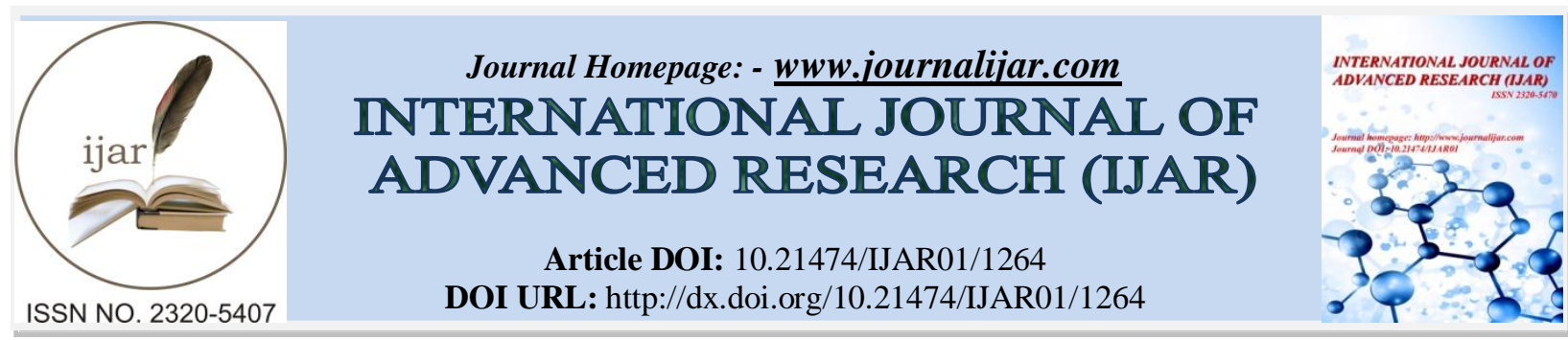

RESEARCH ARTICLE

\title{
Effect of semiorganic additive on the growth and characterization of NLO properties of NaAP single crystals for optoelectronic applications.
}

\section{S. Shek Dhavud ${ }^{1}$ and ${ }^{*}$ J. Thomas Joseph Prakash ${ }^{2}$}

1. PG and Research Department of Physics, Jamal Mohamed College (Affiliated to Bharathidasan University), Trichy-20, Tamilnadu, India.

2. PG and Research Department of Physics, Government Arts College (Affiliated to Bharathidasan University), Trichy-22, Tamilnadu, India.

\section{Manuscript Info}

Manuscript History

Received: 15 June 2016

Final Accepted: 16 July 2016

Published: August 2016

Keywords:-

X-ray diffraction,

Nonlinear optical material,

Dielectric properties,

\begin{abstract}
The pure and semiorganic material of L-Histidine monohydrochloride dihydrate (LHMHC dihydrate) doped sodium acid phthalate (NaAP) single crystals were effectively grown from deionized water by slow evaporation solution technique. Single crystal X-ray diffraction is employed to know the precise information about the crystallographic structure and also the title materials are orthorhombic crystal system with space group B2ab. The interaction of various functional groups was recognized by FT-IR spectral study. The title materials are optically transparent and having a lower cut-off wavelength as $300 \mathrm{~nm}$, thereby confirming the enhancement of nonlinear optical property and optical band gap of the pure and LHMHC dihydrate doped NaAP crystals have been determined as $3.5 \mathrm{eV}$ and $4 \mathrm{eV}$ by UV-Visible studies. The dielectric constant and dielectric loss of the pure and LHMHC dihydrate doped NaAP crystals decrease as the frequency of applied field increases at the end. The mechanical behaviors of the grown crystals were assessed by Vickers microhardness activities. The SHG efficiency of the grown crystals confirms NLO behavior of green colour emission to offer frequency doubling process for photoelectronic applications.
\end{abstract}

Copy Right, IJAR, 2016,. All rights reserved.

\section{Introduction:-}

Derivative of phthalic acids based crystals are potential candidates for nonlinear optical (NLO) and electro-optic processes. The phthalate semiorganic crystals are extensively recognized for their various application in the longwave X-ray spectrometers and piezoelectric, optical, NLO and elastic properties are also investigated in detail [1-2]. Acid phthalate crystals were widely used as substrates for thin film deposition of organic nonlinear materials [3]. In general, Phthalate complexes crystals are being widely examined for their promising electronic and optoelectronic applications. Potassium acid phthalate (KAP), Rubidium acid phthalate (RbAP), Cesium acid phthalate (CsAP), Thallium acid phthalate (TiAP), Lithium acid phthalate (LiAP), Lithium sodium acid phthalate (LiNaP),

Corresponding Author:- J. Thomas Joseph Prakash

Address:- PG and Research Department of Physics, Government Arts College (Affiliated to Bharathidasan University), 
Ammonium acid phthalate $\left(\mathrm{NH}_{4} \mathrm{AP}\right)$ are reported as semiorganic phthalic acid crystals. All these compounds are stabilized by extensive networks of hydrogen bonds.

In this series, sodium hydrogen phthalate $\left[\mathrm{Na}\left(\mathrm{C}_{6} \mathrm{H}_{4} \mathrm{COOH}-\mathrm{COO}\right)\right]$ also called sodium acid phthalate (NaAP) is a semiorganic material that goes to orthorhombic class of alkali acid series, which is having laser-induced damage threshold and the values of SHG efficiency much higher than that of KAP crystal with non-hygroscopic nature [4]. $\mathrm{NaAP}$ consists of $\mathrm{Na}$ ions, phthalate ions and water molecules. The $\mathrm{Na}^{+}$ion is surrounded by six $\mathrm{O}$ atoms, four from the ionizing carboxyl groups, one from an unionized carboxyl group and one from the water molecule. NaAP crystallizes in an orthorhombic symmetry with the space group B2ab [5]. There are only a few reports available on the effect of amino acid on the NLO efficiency of NaAP crystals.

In the present manuscript, the effect of L-Histidine monohydrochloride dihydrate on the growth and properties of Sodium Acid Phthalate single crystals. The results of the characterization studies conclude that the amino acid (L-Histidine monohydrochloride dihydrate ) dopant play a significant role in the viewpoint of optoelectronic device applications.

\section{Experimental:-}

Pure NaAP crystal was synthesized by taking sodium bicarbonate (Merck, AR grade, 99.8\%) and phthalic acid (Merck, AR grade, $99 \%$ ) in an equimolar ratio and dissolved in deionized water. The solution was thoroughly stirred for 3 hrs continuously using a magnetic stirrer till to get the homogeneous solution and the saturated solution was filtered using filter paper and it was kept in undisturbed conditions. The quality of the grown crystals was enhanced by recrystallization process. After a period of 35 days, transparent good quality of the crystal of pure NaAP was harvested (Fig. 1a).

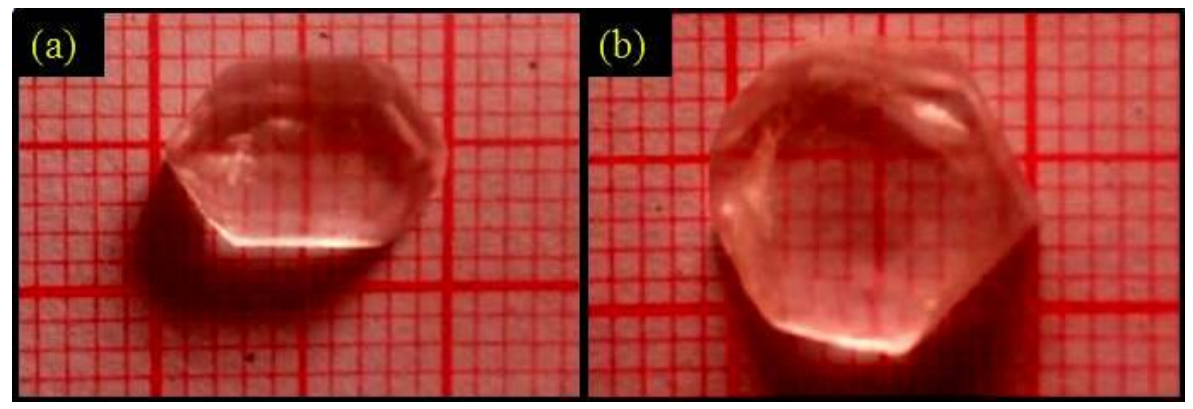

Figure 1a:- As grown crystal of the pure NaAP. Figure 1b: As grown crystal of LHMHC dihydrate doped NaAP crystal.

0.5 Mole \% of L-Histidine monohydrochloride (Loba, AR grade, 99\%) was added to the mother solution of NaAP and the solution was thoroughly stirred endlessly for $4 \mathrm{hrs}$ till to get the homogeneous solution. The saturated solution was filtered using filter paper and it was kept in undisturbed conditions. After a period of 47 days, transparent good quality of the crystal of LHMHC dihydrate doped NaAP was harvested (Fig. 1b). The grown crystals are optically transparent and non-hygroscopic. The pronounced distinction between NaAP and KAP crystal is that NaAP grows more uniformly in all three axes when compared to KAP crystals. This is a significant factor while fabricating devices for SHG applications.

\section{Results and Discussion:-}

Single crystal $\mathrm{X}$-ray diffraction analysis:-

The pure and LHMHC dihydrate doped NaAP crystals were subjected to single crystal X-ray diffraction analyses using ENRAFNONIUS CAD 4 diffractometer with Mo $\mathrm{K} \alpha$ radiation $(\lambda=0.71073 \AA)$ to identify the unit cell parameters. The observed lattice parameter values of pure and LHMHC dihydrate doped NaAP crystals are found to be $a=6.75 \AA, b=9.33 \AA, c=26.65 \AA, \alpha=\beta=\gamma=90^{\circ}$ and the volume of the unit cell was found to be $1678 \AA^{3}$ for pure NaAP crystal and $a=6.77 \AA, b=9.38 \AA, c=26.75 \AA$, for LHMHC dihydrate doped NaAP crystal, the volume of the unit cell was found to be $1699 \AA^{3}$ and the grown crystals belong to the space group B2ab which is in good agreement with the reported values $[4,6]$ of pure NaAP crystal. The slight variations in the cell parameters indicated the incorporation of LHMHC dihydrate into the crystal lattice of pure NaAP crystal. 


\section{Functional group analysis:-}

The grown crystals were subjected to FT-IR analysis by Perkin Elmer RXI FTIR spectrometer by KBr pellet technique in the wavelength range between 400 and $4000 \mathrm{~cm}^{-1}$. FT-IR spectra of pure and LHMHC dihydrate doped NaAP crystals was recorded as shown in Fig.2a and Fig.2b to confirm and see the effect of doing on functional groups of the parent compound.

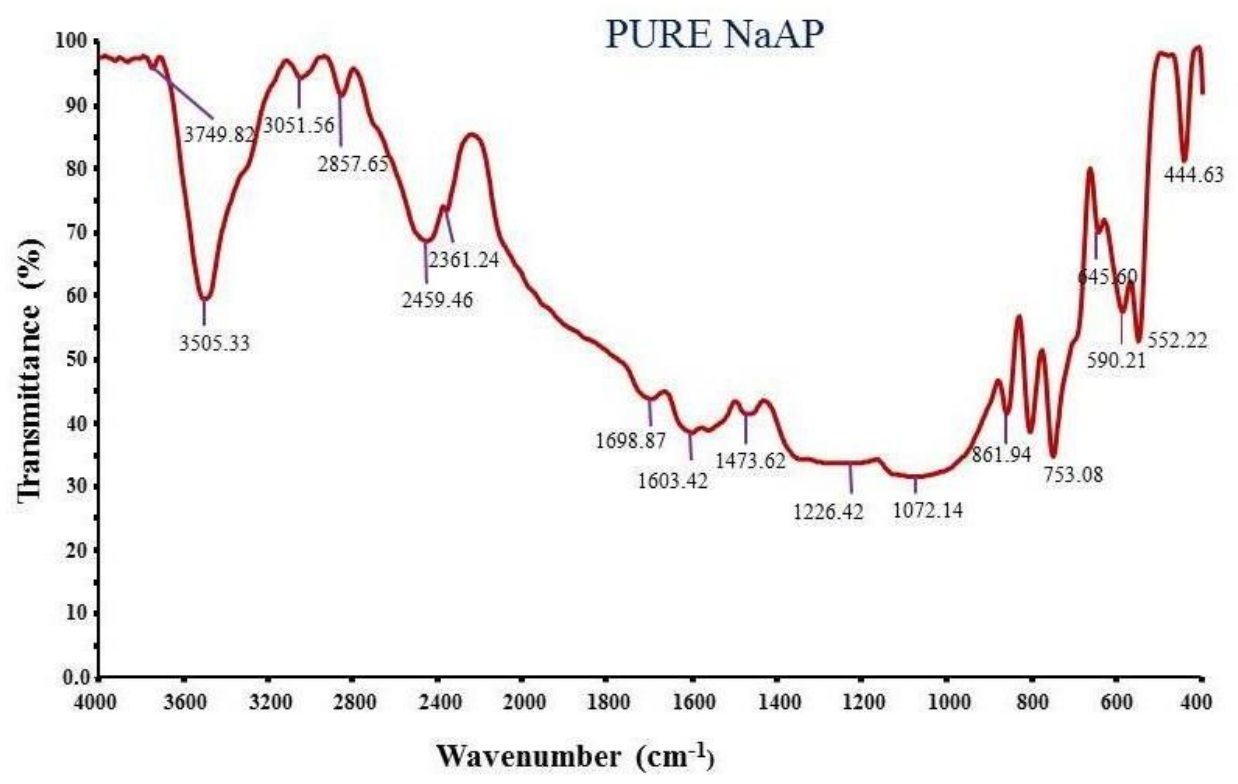

Figure 2a: FTIR spectrum of pure NaAP crystal

LHMHC DIHYDRATE DOPED NaAP

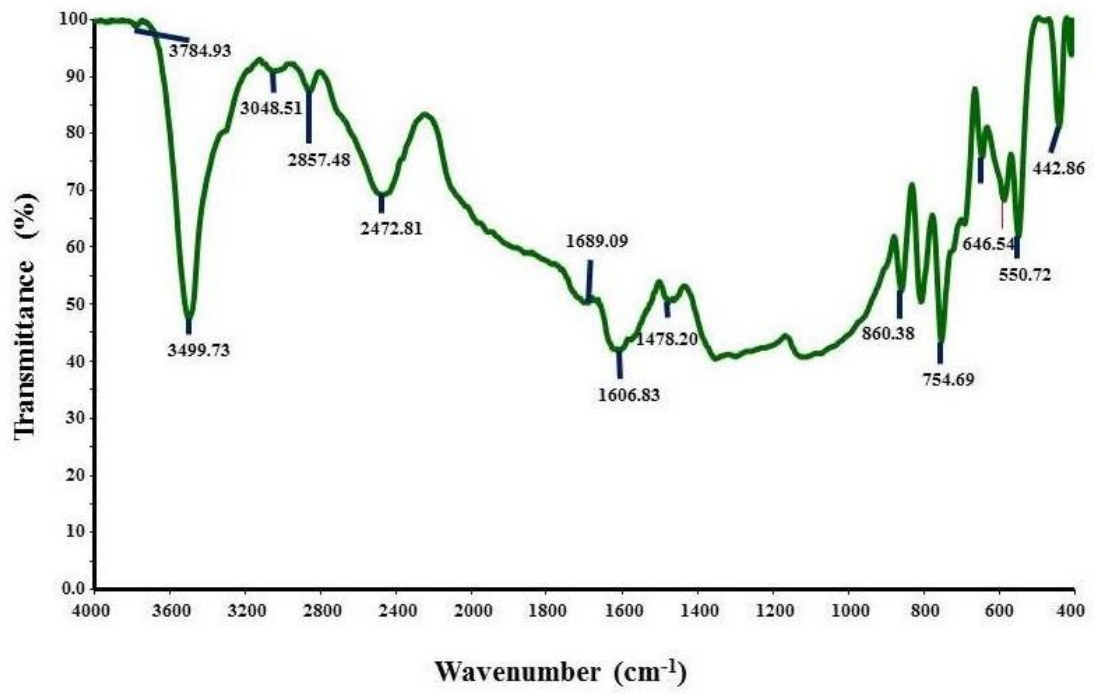

Figure 2b: FTIR spectrum of LHMHC dihydrate doped NaAP crystal

The O-H stretching vibration has more sensitive to the hydrogen bond interaction with the amino acid dopants [7]. The observed bands and their corresponding assignments are presented in Table 1. 
Table 1: Observed FTIR bands of the grown crystals and their assignments

\begin{tabular}{|c|c|c|}
\hline \multicolumn{2}{|c|}{ Observed wavenumber $\left(\mathrm{cm}^{-1}\right)$} & \multirow[b]{2}{*}{ Assignments } \\
\hline Pure NaAP & $\begin{array}{l}\text { LHMHC Dihydrate } \\
\text { doped NaAP }\end{array}$ & \\
\hline 3505.33 & 3499.73 & -O-H stretching, intermolecular bonded $\mathrm{OH}$ \\
\hline 3051.56 & 3048.51 & -O-H bending, $\mathrm{CH}$-bending in aromatic ring \\
\hline 2857.65 & 2852.48 & C-H-bending-asymmetric \\
\hline 2459.46 & 2472.81 & C-H-bending \\
\hline 1698.87 & 1689.09 & $\mathrm{C}=\mathrm{O}$ vibration (Carboxyl group) \\
\hline $1473.62 \& 1603.42$ & $1478.20 \& 1606.83$ & C-C skeletal aromatic ring vibration \\
\hline 861.94 & 866.38 & $\mathrm{C}-\mathrm{H}$ out of plane bending \\
\hline 753.08 & 758.69 & C-C stretching \\
\hline 645.60 & 646.54 & $\mathrm{C}$-O wagging \\
\hline 552.22 & 550.72 & $\mathrm{C}=\mathrm{C}-\mathrm{C}$ out of plane ring deformation \\
\hline 444.63 & 442.86 & $\mathrm{C}=\mathrm{C}$ plane bending \\
\hline
\end{tabular}

From Table-1, the absorption peaks observed around $3505 \mathrm{~cm}^{-1}$ corresponds to the $\mathrm{O}-\mathrm{H}$ stretching vibration of the carboxylic acid for pure NaAP crystal whereas in the case of LHMHC dihydrate doped NaAP crystal, these peaks gets shifted towards the lower frequency and the absorption peak is observed around $3499 \mathrm{~cm}^{-1}$ showing a red shift in the value. The absorption peak around $1698 \mathrm{~cm}^{-1}$ corresponds to the $-\mathrm{C}=\mathrm{O}$ carboxylate ion symmetric stretching of $=\mathrm{O}$ for pure NaAP. In the case of LHMHC dihydrate doped NaAP, the absorption value shows a blue shift by shifting the peak to $1689 \mathrm{~cm}^{-1}$. A marked enhance in the carboxylate ion and the carboxylic acid stretching was observed in LHMHC dihydrate doped NaAP when compared to pure NaAP which is due to the influence of dopant. Carboxylic stretching vibration is recognized to produce resolved multiple bands between $2500 \mathrm{~cm}^{-1}$ and $3500 \mathrm{~cm}^{-1}$. The difference in sharpness of multiple bands may be taken as an evidence for the doping of LHMHC dihydrate doped NaAP. The absorption peaks obtained at $1473 \mathrm{~cm}^{-1}$ and $1603 \mathrm{~cm}^{-1}$ for pure NaAP whereas $1478 \mathrm{~cm}^{-1}$ and $1606 \mathrm{~cm}^{-1}$ for LHMHC dihydrate doped NaAP corresponds to C-C skeletal aromatic ring vibration. Apart from this, some bending vibrations and a wagging vibration take place owing to the presence of the carboxylate group. The wagging vibration occurs at $645 \mathrm{~cm}^{-1}$ and $646 \mathrm{~cm}^{-1}$ corresponds to the C-O wagging for pure and LHMHC dihydrate doped NaAP. The vibrations occurring at $861 \mathrm{~cm}^{-1}, 860 \mathrm{~cm}^{-1}$ and $444 \mathrm{~cm}^{-1}$ and $442 \mathrm{~cm}^{-1}$ absorption peaks is ascribed to the $\mathrm{C}-\mathrm{H}$ out of plane bending and $\mathrm{C}=\mathrm{C}$ plane bending for pure and LHMHC dihydrate doped NaAP. One $\mathrm{C}=\mathrm{C}-\mathrm{C}$ out of plane ring deformation vibration occurs at the $552 \mathrm{~cm}^{-1}$ and $550 \mathrm{~cm}^{-1}$ for pure and LHMHC dihydrate doped NaAP. C-C stretching vibration occurs at $753 \mathrm{~cm}^{-1}$ and $754 \mathrm{~cm}^{-1}$ for pure and LHMHC dihydrate doped NaAP. In the case of dopants, the same peaks were observed with some slight shifts in the undoped spectrum. All these bond assignments are in excellent agreement with that of the reported values [4]. Here the shifting of peaks denotes that LHMHC dihydrate doped NaAP is added into the crystal lattice of pure NaAP successfully. Hence, the bonding interaction between NaAP and LHMHC dihydrate doped NaAP are clearly established in the FTIR analysis. 


\section{Linear optical analysis:-}

The optical transmission spectrum of pure and LHMHC dihydrate doped NaAP crystals were recorded in the wavelength region from 190-1100 $\mathrm{nm}$ using PERKIN ELMER LAMBDA 35 UV-Visible spectrophotometer (Fig.3).

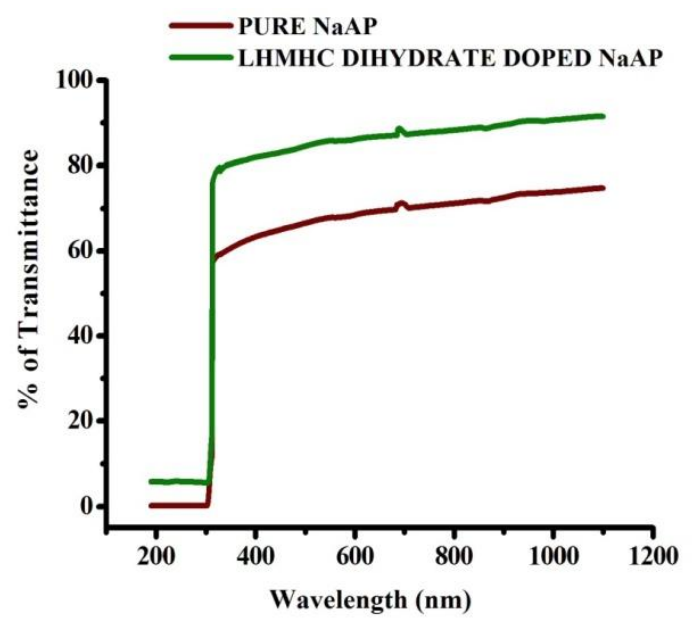

Figure 3: UV-Visible transmission spectrum of pure and LHMHC dihydrate doped NaAP crystals

For optical fabrication, the crystal should be highly transparent in the significant region of wavelength [8]. From Fig.3, it shows that the transparency of the grown crystals is good in the entire UV-Visible region and it is evident that the title compound has a low cut-off wavelength at $300 \mathrm{~nm}$ which is appropriate for SHG laser radiation of $1064 \mathrm{~nm}$ for frequency doubling process. Pure NaAP crystal has $60 \%$ transmittance while LHMHC dihydrate doped NaAP crystal has $80 \%$ transmittance in the entire UV-Visible region. The obtained results were compared with the reported work [9] and it was observed that LHMHC dihydrate doped NaAP crystal show better optical transmittance value than L-Valine doped NaAP crystal in the entire visible region. The increment in the optical transmittance of LHMHC dihydrate doped NaAP crystal compared to pure NaAP one suggests the enhancement in optical quality. As a result, LHMHC dihydrate doped NaAP crystal are more suitable for electro-optic modulation and are a potential candidate for NLO applications. The optical band gap (Eg) of the grown crystals has been calculated using the formula [10]

$\alpha h \gamma=A\left(E_{\mathrm{g}}-h \gamma\right)^{\mathrm{n}} \quad(\mathrm{n}=1 / 2$ for direct allowed transition $)$

Where, $\alpha$ is optical absorption coefficient and $h y$ is the photon energy. ' $\mathrm{A}$ ' is a slope of the Tauch edge and it is called the band tailing parameter which depends on the width of localized states in the band gap. The value of energy band gap is estimated from the graph drawn between $(\alpha h y)^{2}$ versus photon energy (hy) as shown in Fig.4. 


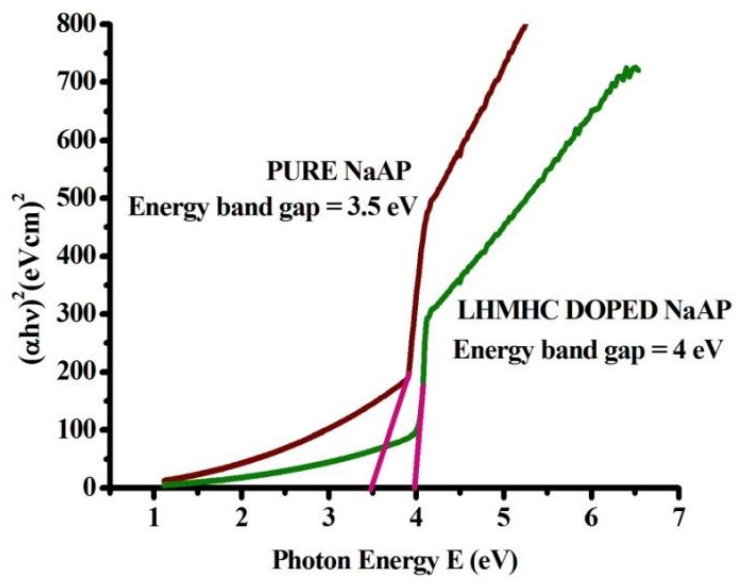

Figure 4: Plot of $(\alpha h \gamma)^{2}$ versus photon energy (E) in electron volt for pure and LHMHC dihydrate doped NaAP crystals.

The energy band gap is solved by extrapolating the linear part from the maximum absorption end to $h y$ axis. The intersecting point on photon energy axis is band gap energy of grown crystals. The energy band gap of pure NaAP crystal has been found to be $3.5 \mathrm{eV}$ while LHMHC dihydrate doped NaAP has been found to be $4 \mathrm{eV}$. Because of a large band gap of LHMHC dihydrate doped NaAP $(4 \mathrm{eV})$, this crystal can be an appropriate compound for the optoelectronic devices such as LED and Laser diodes [11].

The extinction coefficient could be a measure of the amount of loss of electromagnetic radiation through scattering and absorption of grown crystals per unit thickness. Extinction coefficient value $(\mathrm{K})$ has been calculated using the following equation

$K=\lambda \alpha / 4 \pi$

Where, absorption coefficient $(\alpha)$ is calculated from the transmittance graph by the equation

$\alpha=2.303 \log (1 / \mathrm{T}) / \mathrm{d}$

' $\mathrm{d}$ ' is the thickness of the sample $(2 \mathrm{~mm})$ and ' $\mathrm{T}$ ' is the transmittance of the sample. The extinction coefficient values of the grown crystals are decreasing with respect to wavelength as shown in Fig. 5.

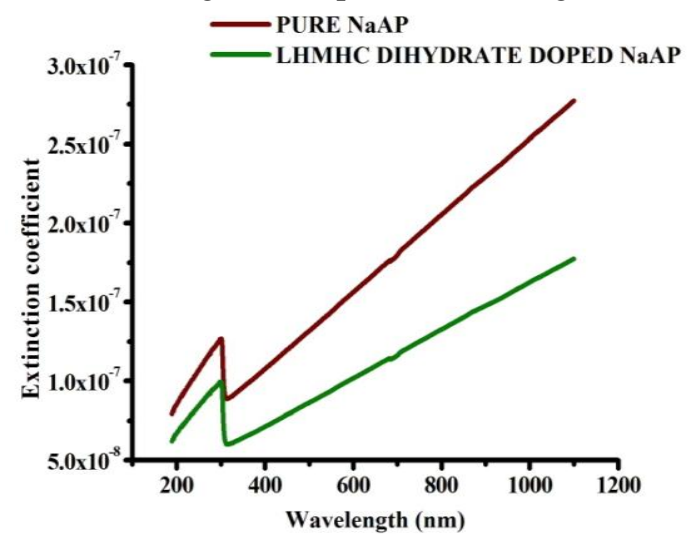

Figure 5: Plot of extinction coefficient versus wavelength for pure and LHMHC dehydrate doped NaAP crystals

Fig.6a \& $6 \mathrm{~b}$ show that the variation of optical reflectance of pure and LHMHC dihydrate doped NaAP crystals with optical absorption coefficient. When optical reflectance increases, optical absorption coefficient also increases exponentially. The variation is in keeping with the correction between optical reflectance and optical absorption coefficient represented by the following equation. 


$$
=\frac{1 \pm \sqrt{(1-\exp (-\operatorname{ad})+\exp (a d))}}{1+\exp (-\operatorname{ad})}
$$

The maximum value of the optical reflectance for pure NaAP is 88.5 when the optical absorption coefficient is 4500 while the optical reflectance for LHMHC dihydrate doped NaAP is 58 when the optical absorption coefficient is 2000. The refractive index of optical materials is notably significant for the applications in integrated optic devices (switches, filters, modulators, etc.) [12].
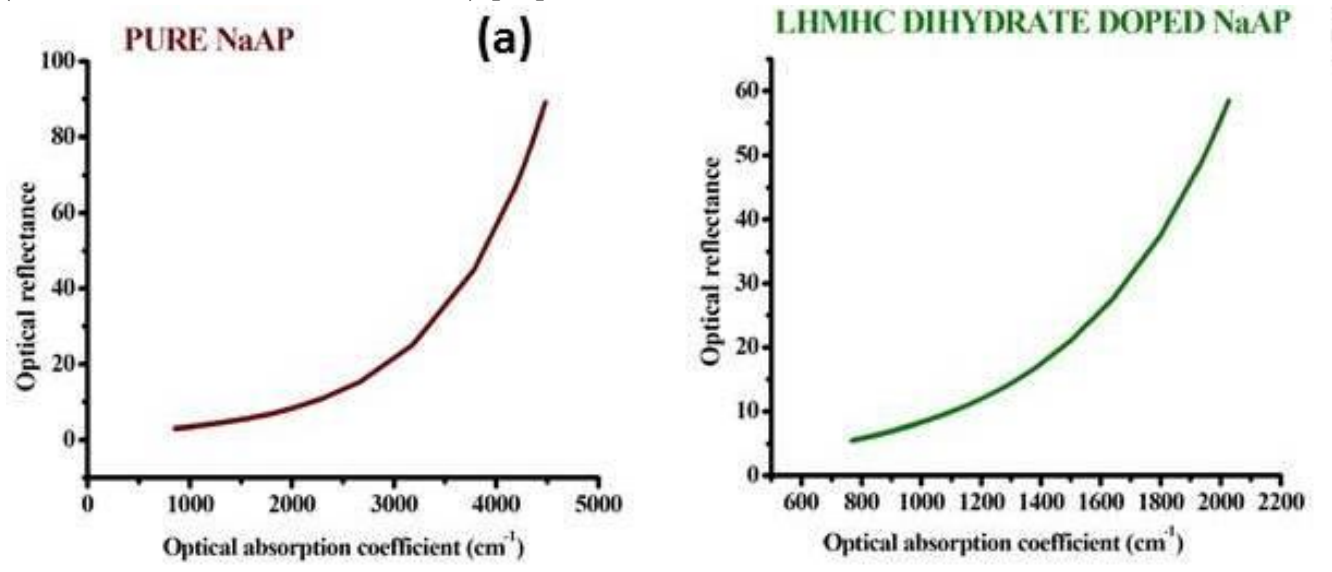

(b)

Figure 6a: Plot of reflectance versus wavelength for pure NaAP crystal

Figure 6b: Plot of reflectance versus wavelength for LHMHC dihydrate doped NaAP crystal

The refractive index (n) of the grown crystals are calculated from the equation below

$n=\frac{-(R+1) \pm \sqrt{-a R^{2}+10 R-a}}{2(R-1)}$

From Fig.7, the refractive index of the grown crystals increases with increasing wavelength and then decreases when reaching a maximum value in the wavelength $1100 \mathrm{~nm}$. The refractive index of the pure NaAP at $1100 \mathrm{~nm}$ was determined to be 1.75 while the refractive index of the LHMHC dihydrate doped NaAP at $1100 \mathrm{~nm}$ was found to be 1.46. The high transmission, low refractive index and low reflectance of LHMHC dihydrate doped NaAP crystal makes a suitable material for nonlinear optical device applications and antireflection coating in solar thermal and [13]. Hence, this material could prove effective in the fabrication of optoelectronic devices.

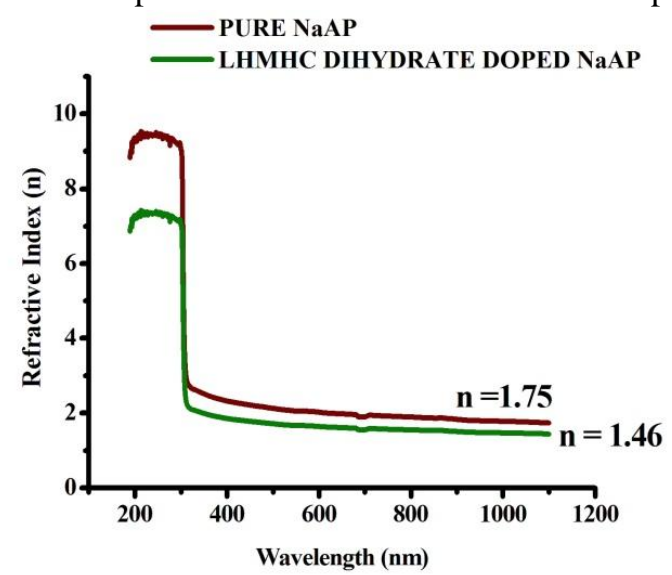

Figure 7:- Plot of refractive Index versus wavelength for pure and LHMHC dihydrate doped NaAP crystals 


\section{Dielectric analysis:-}

The dielectric characteristics of the material are important to examine the lattice dynamics in the crystal. So, the grown crystals were subjected to dielectric studies using HP 4275 multi- frequency LCR meter. The well-polished single crystals of pure and LHMHC dihydrate doped NaAP were employed for dielectric studies. The surface of the grown crystals was an electrode with a silver paste for electrical contact. The experiment was accomplished for the frequencies from $50 \mathrm{~Hz}$ to $1 \mathrm{MHz}$. The dielectric constant $\left(\varepsilon_{y}\right)$ was calculated by the equation,

$$
\varepsilon_{\mathrm{T}}=\frac{\mathrm{Cd}}{\mathrm{s}_{\mathrm{o}} \mathrm{A}}
$$

Where $\mathrm{C}$ is the capacitance, $\mathrm{d}$ is the thickness of the crystal, $\varepsilon_{\mathrm{o}}$ is the permittivity of free space and $\mathrm{A}$ is the area of the crystal $\left(2.83 \times 10^{-5}\right)$. The dielectric loss was calculated by the relation,

$$
\varepsilon^{\mp}=\varepsilon_{\mathrm{Y}} D
$$

Where, D is the dissipation factor. Fig. $8 \mathrm{a} \&$ Fig. $8 \mathrm{~b}$ show the variation of the dielectric constant and dielectric loss of the pure and LHMHC dihydrate doped NaAP crystals with frequency.
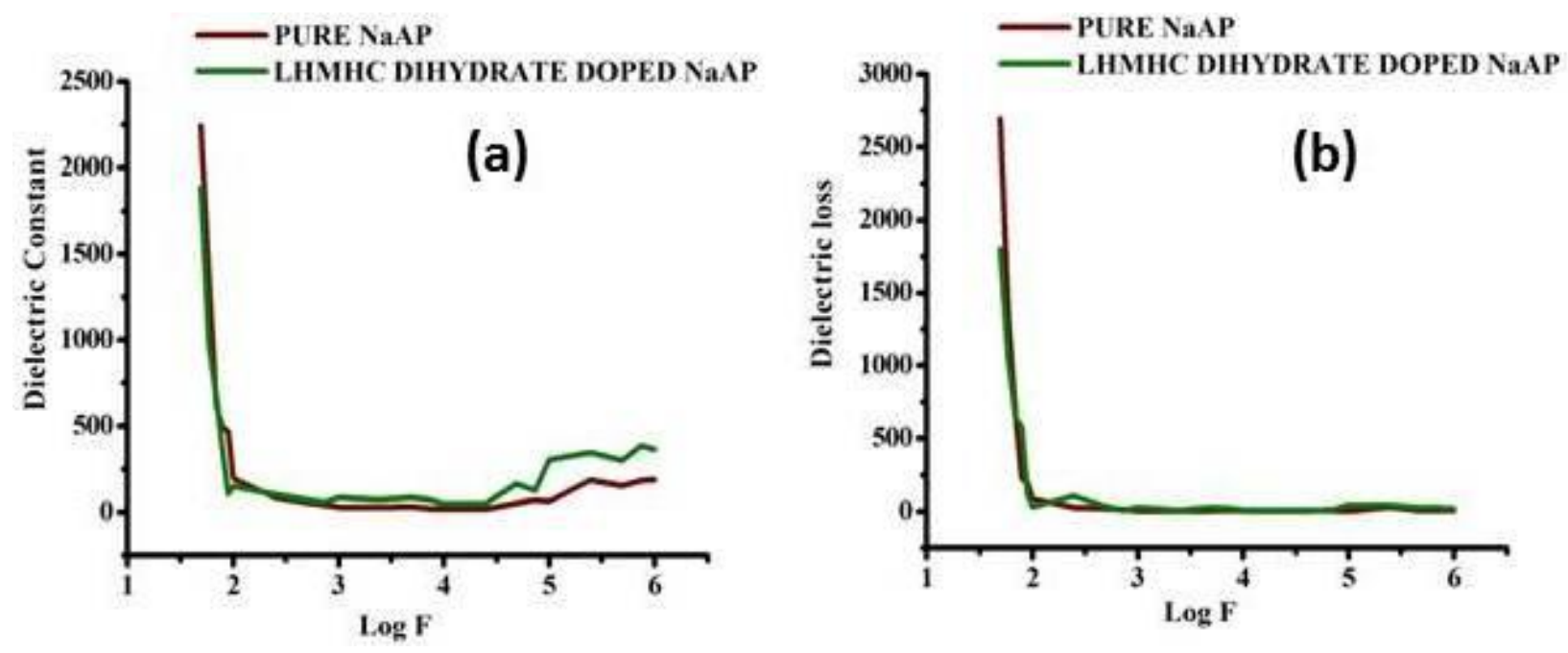

Figure 8a:- Plot of dielectric constant vs Log F for pure and LHMHC dihydrate doped NaAP crystals. Figure 8b:- Plot of dielectric loss vs Log F for pure and LHMHC dihydrate doped NaAP crystals.

From figures $8 \mathrm{a} \& 8 \mathrm{~b}$, it is observed that the values of dielectric constant and dielectric loss of the pure and LHMHC dihydrate doped NaAP crystals decreases with increases in frequency and attain saturation at higher frequencies. The dielectric constant with very high value at low frequencies is due to different polarizations namely ionic, electronic, orientation and space charge [14]. The low dielectric constant at high frequency is attributed to space charge polarization and it is significant for the construction of photonic and NLO devices. The dielectric loss values are high at lower frequencies and they are low at higher frequencies. The low dielectric loss with higher frequencies for LHMHC dihydrate doped NaAP crystal compare to pure NaAP possesses enhanced optical quality with lesser defects and this shows that LHMHC dihydrate doped NaAP crystal can be used for optoelectronic device applications.

\section{Mechanical stability analysis:-}

The mechanical studies of the grown crystals were made by Vickers microhardness activity at room temperature. Crystals which is free from cracks with flat and smooth surfaces were chosen for the static indentation examinations. The crystal was fixed properly on the base of the microscope. Now, the selected smooth faces of the grown crystals were indented gently by varying loads from $20 \mathrm{~g}$ to $100 \mathrm{~g}$ for a period of $10 \mathrm{~s}$ by Vickers diamond indenter joined to an incident ray analysis microscope and the length of the two diagonals of diamond indenter was determined by a 
calibrated micrometer joined to the eyepiece of the microscope. For a particular load, three well-defined indentations were measured and the average value (d) was calculated. The Vickers hardness $\left(\mathrm{H}_{\mathrm{v}}\right)$ numbers were calculated for different loads [15] using the following relation.

$\mathrm{H}_{\mathrm{v}}=(1.8544 \times \mathrm{P}) / \mathrm{d}^{2} \mathrm{~kg} / \mathrm{mm}^{2}$

Where ' $\mathrm{P}$ ' is the applied load in kilogram and ' $\mathrm{d}$ ' is the average or mean diagonal length of the indentation marks. The result is plotted (Fig. 9).

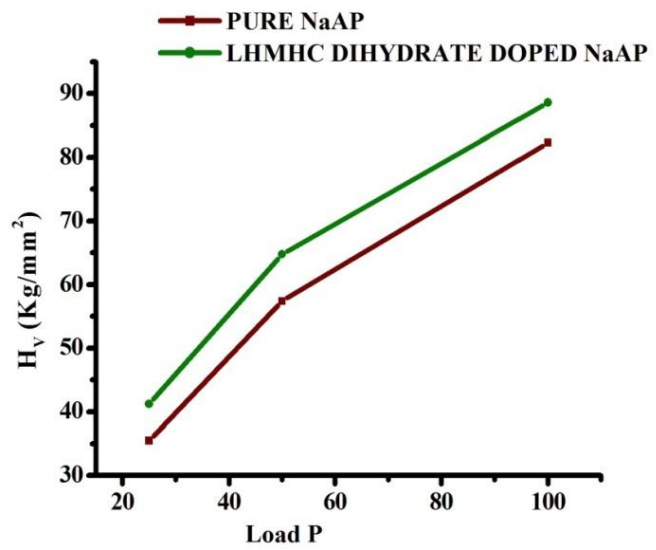

Figure 9:- Variation of Hv with applied load for pure and LHMHC dihydrate doped NaAP crystals.

The grown crystals show the reverse indentation size effect in which the hardness values increases with increasing the applied load [16, 17]. Crack initiation and materials chipping become important beyond $100 \mathrm{~g}$ of the applied load. Hence, hardness experiment could not be done above this load. It shows that the density of defects in the crystal is in the form of vacancy [18]. Increasing noncentrosymmetric due to the presence of an increased number of vacancies could also be the physical origin of the observed phenomenon. Meyer's law relates load and size of indentation as

$\mathrm{P}=\mathrm{k}_{1} \mathrm{~d}^{\mathrm{n}}$

Where ' $k_{1}$ ' is the material constant, ' $n$ ' is the work hardening coefficient. Taking logarithm on both sides of Eq.(9), we get

$\log \mathrm{P}=\log \mathrm{k}_{1}+\mathrm{n} \log \mathrm{d}$

The plot of ' $\log$ P' against ' $\log$ d' is shown in Fig. 10a \& Fig. 10b which provides a straight line (after least square fitting). The slope of the line provides ' $n$ ' and it is found to be 4.4 for pure NaAP crystal and 2.8 for LHMHC dihydrate doped NaAP crystal. According to Onitsch [19], ' $\mathrm{n}$ ' should lie between 1 and 1.6 for hard materials and must be above 1.6 for softer materials. Hence, the grown crystals belong to a softer material category.

(a)
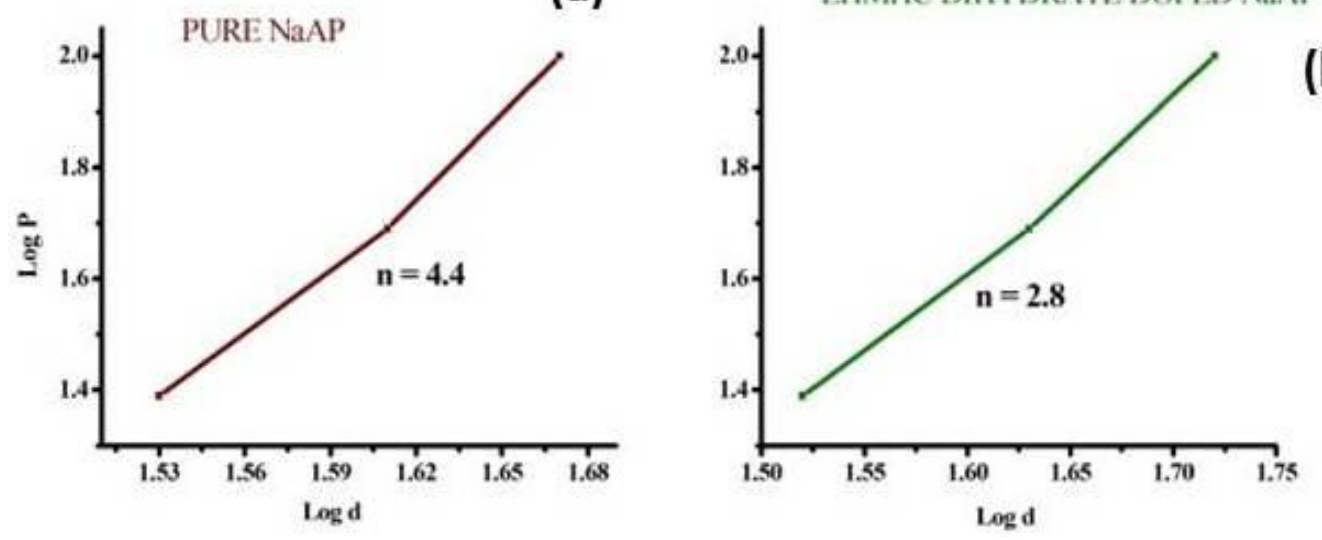

(b)

Figure 10a:- Plot of $\log \mathrm{P}$ versus $\log \mathrm{d}$ for pure NaAP crystal.

Figure 10b: Plot of $\log \mathrm{P}$ versus $\log \mathrm{d}$ for LHMHC dihydrate doped NaAP crystal 


\section{Second Harmonic Generation efficiency measurements:-}

The Second Harmonic Generation property of the grown crystals was tested by the Kurtz-Perry powder method [20]. The emission of green radiation from the samples confirms the SHG in the crystal. The result obtained for pure NaAP crystal shows a powder SHG efficiency of about 1.53 times that of KDP crystal and 1.78 times that of KDP for LHMHC dihydrate doped NaAP crystal. The intensity of the green light from several points on the powdered sample shows that the slight increase in SHG signal intensity for LHMHC dihydrate doped NaAP crystal as compared to pure NaAP crystal and this may be due to central ion in metal organic complex gives a certain anisotropic field to keep NLO active chromophores which drastically vary the hyperpolarizability [21].

\section{Conclusion:-}

Transparent single crystals of pure and LHMHC dihydrate doped NaAP were successfully grown from aqueous solution by low temperature slow evaporation solution growth technique. The crystal system of the grown crystals was confirmed by the single crystal XRD studies and the presence of dopants in the crystal lattice of the pure NaAP slightly changed the lattice parameters without affecting the basic structure. FTIR trace revealed the presence of amino groups and functional groups. UV-Visible spectrum shows that by doping LHMHC dihydrate doped NaAP the percentage of transmission is increased. Significant larger optical transparency and lower cut-off value down to $300 \mathrm{~nm}$ make it a promising material for NLO applications containing frequency doubling process. The dielectric constant and dielectric loss with low values at high frequencies show the high optical quality of the grown crystals with lesser defects which are the desirable property for applications in photonic and optoelectronic devices. The SHG efficiency of LHMHC dihydrate doped NaAP has enhanced than pure NaAP. Vickers microhardness test suggests that the grown crystals belong to a soft material category. Owing to the wide optical transparency, high mechanical stability and relatively high SHG efficiency of LHMHC dihydrate doped NaAP crystal is a potential candidate for fabrication of optoelectronic devices and laser applications.

\section{Acknowledgment:-}

The authors are grateful to Dr. Babu Varghese, Head, SAIF IIT-Madras, B.S. Abdur Rahman University, Chennai and St. Joseph's College, Trichy for providing characterization facilities.

\section{References:-}

1. C. Ecolivet, A. Miniewicz, M. Sanquer (1992). Study of elastic properties of sodium, potassium and rubidium acid phthalates by brillouin scattering, J. Phys.Chem.Solids. 53, 511-520.

2. N. Kejalakshmy, K. Srinivasan (2004). Growth, optical and electro-optical characterizations of potassium hydrogen phthalate crystals doped with $\mathrm{Fe}^{3+}$ and $\mathrm{Cr}^{3+}$ ions, Opt. Mater. 27, 389-394.

3. W. Sander Graswinckel, Fieke J. Van den Bruele, Willem J. P. Van Enckevort, Elias Vlieg (2007). Epitaxy of organic crystal films:Phenanthrene on Potassium Acid Phthalate, Cryst. Growth Des. 7, 243-249.

4. R. Bairava Ganesh, V. Kannan, K. Meera, N.P. Rajesh, P. Ramasamy (2005). Synthesis, growth and characterization of a new nonlinear optical crystal sodium acid phthalate, J. Crystal growth. 282, 429-433.

5. R.A. Smith (1975). The crystal structure of sodium acid phthalate hemihydrate, Acta Cryst. B31, 2345-2347.

6. S. Krishnan, C. Justin Raj, S. Dinakaran, R. Uthrakumar, R. Robert, S. Jerome Das (2008). Optical, thermal, dielectric and ferroelectric behavior of sodium acid phthalate (SAP) single crystals,

J. Phys. Chem. Solids. 69, 2883-2887.

7. T.Baraniraj, P.Philominathan (2011). Growth, thermal, mechanical and dielectric studies of glycine doped Potassium Acid Phthalate single crystals, J.Miner.Mater.Char and Engg. 10, 805-815.

8. V. Krishnakumar, R. Nagalakshmi (2005). Crystal growth and vibrational spectroscopic studies of the semiorganic non-linear optical crystal - bisthiourea zinc chloride, Spectrochim. Acta, Part A. 61, 499-507.

9. L. Ruby Nirmala, J. Thomas Joseph Prakash (2013). Effect of L-Valine on the growth and characterization of Sodium Acid Phthalate (SAP) single crystals, Spectrochim. Acta. A. 110, 425 - 429.

10. J. Tauc (1970). The Optical Properties of Solids (North-Holland, Amsterdam).

11. K. Kumar, K. Ramamoorthy, P.M. Koinkar, R. Chandramohan, K. Sankaranarayan (2006). A novel in situ synthesis and growth of $\mathrm{ZnAl}_{2} \mathrm{O}_{4}$ thin films, J. Crystal growth. 289, 405-407.

12. M.A.M. Khan, M.W. Khan, M. Alhoshan, M.S. Alsalhi, A.S. Aldwayyan (2010). Influences of Co doping on the structural and optical properties of $\mathrm{ZnO}$ nanostructured, Appl Phys A. 100, 45-51.

13. R. Santhakumari, K. Ramamurthi (2011). Structural, thermal and optical characterization 
of an organic NLO material - Benzaldehyde thiosemicarbazone monohydrate single crystals, Spectrochim Acta A, 78, 653-659.

14. K.V. Rao, A. Smakula (1965). Dielectric properties of cobalt oxide, Nickel oxide and their mixed crystals, J. Appl. Phys, 36, 2031-2038.

15. J. Bowman, M. Bevis (1977). The evaluation of the structure and hardness of processed plastics by the Vickers microhardness test, Colloid Pom. Sci, 255, 954-966.

16. J. Gong, Y. Li (2000). An energy-balance analysis for the size effect in low-load hardness testing, J. Mater. Sci, 35, 209-213.

17. S. Karan, S.P.S. Gupta (2005). Vickers microhardness studies on zinc sulphate heptahydrate, Mater. Sci. Eng, A398, 198-203.

18. I.V. Kityk, B. Marciniak (2001). A. Mefleh, Photoinduced second harmonic generation in molecular crystals caused by defects, J.Phys. D: Appl. Phys, 34, 1-4.

19. E.M. Onitsch (1947). Über die mikrohärte der metalle, Mikroscopia, 2, 131-151.

20. S.K. Kurtz, T.T. Perry (1968). A Powder Technique for the evaluation of nonlinear optical materials, J. Appld. Phys, 39, 3798-3812.

21. Robert C. Miller (1964). Optical second harmonic generation in piezoelectric crystals, Appl. Phys. Lett, 5, 17-19. 